\title{
Spectral Measurement of Photon Emission from Individual Gold Nanoparticles Using Scanning Tunneling Microscopy
}

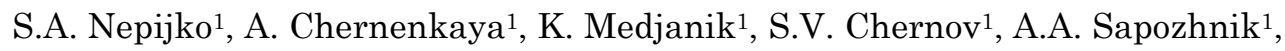 \\ L.V. Odnodvorets ${ }^{2}$, I.Yu. Protsenko ${ }^{2}$, W. Schulze ${ }^{3}$, G. Ertl ${ }^{3}$, G. Schönhense ${ }^{1}$ \\ ${ }^{1}$ Institute of Physics, University of Mainz, 7, Staudingerweg, 55128 Mainz, Germany \\ 2 Sumy State University, 2, Rymskogo-Korsakova St., 40007 Sumy, Ukraine \\ ${ }^{3}$ Fritz Haber Institute of the Max Planck Society, 4-6, Faradayweg, 14195 Berlin, Germany
}

(Received 19 May 2016; revised manuscript received 10 June 2016; published online 21 June 2016)

\begin{abstract}
The light emission spectra of individual Au nanoparticles induced by a scanning tunneling microscope (STM) have been investigated. Two-dimensional ensembles of tunnel-coupled Au particles were prepared by thermal evaporation onto a native oxide silicon wafer in ultrahigh vacuum $\left(10^{-9} \mathrm{mbar}\right)$. Our STM measurements show a single peak at photon energy $1.6 \mathrm{eV}$ in the tunneling mode and two peaks at $2.2 \mathrm{eV}$ (connected with the Mie plasmon) and $1.45 \mathrm{eV}$ (a new peak which was not discussed in literature before) in the field emission mode.
\end{abstract}

Keyworld: Electron-photon spectroscopy, Gold nanoparticle, Scanning tunneling microscope (STM).

\section{INTRODUCTION}

Spectroscopic measurements that allow characterization of nanoparticles as well as the study of their electronic structure (electron density of states near the Fermi level) can be performed in a scanning tunneling microscope (STM). One more possibility for electronic structure investigations corresponds to photon emission in such studies [1-16]. The light emission appears due to (i) inelastic tunneling of some electrons between the tip and the sample (at a tip voltage of a few volts of both polarities) or (ii) electron bombardment of the sample by field emission (when the negative voltage on the tip is about ten volts and higher). This light emission is characterized by a spectrum whose structure is caused mainly by the inter-band transitions, including surface states and the plasmons decay. At the same time the plasmon frequency is determined by the material, size, shape of the nanoparticles and the distances between them as well as the dielectric surrounding. The electronic structure is also deformed by the particle size. The tip material and shape influence the spectrum $[8,9]$. Thus, measurements of the light emission spectra in research of nanoscale objects using a STM have a very high information content. The central point of this method is that it provides the results which are not averaged over a large number of particles with different sizes, i.e. measurements are not integral (like in most optical experiments), but give information about each individual particle [9-14], see also [15, 16].

The measurements were performed on a gold ( $\mathrm{Au})$ nanoparticle film, namely a two-dimensional ensemble of tunnel-coupled $\mathrm{Au}$ nanoparticles on a dielectric substrate. Au was chosen as an object of investigations due to its chemical inertness. In addition, there is a possibility to compare our results of photon emission measurements of Au nanoparticles induced by an STM with previously obtained results for bulk Au single crystals $[2,3,7,8]$.

\section{EXPERIMENTAL DETAILS}

Sample preparation, analysis and measurements were carried out in ultra-high vacuum (UHV) at $10^{-9}$ mbar. Au nanoparticle films were prepared by thermal deposition on a silicon wafer ( $n$-type Si, $\rho \sim 0.01 \Omega \cdot \mathrm{cm}$ ). The Volmer-Weber (3D) growth mechanism is implemented in this system. The substrate cleaning (flash method) as well as Au deposition was performed in the preparation chamber. Resistance of the film was monitored during the growth, deposition was stopped, when the resistance per square reached tens of megaohm, in this case, tunnel coupling is realized between the nanoparticles $[17,18]$. Then the sample was moved into the STM in the main chamber using a transfer system. The STM tip was made of a polycrystalline tungsten (W) wire with diameter of $0.25 \mathrm{~mm}$ by electrochemical etching. In the experiment photon emission of individual Au nanoparticles was obtained using the STM in the tunnel and field emission modes. In order to obtain a large acceptance angle for the light collection, the STM was surrounded by a parabolic mirror. Outside the vacuum chamber the light was focused onto the entrance slit of a spectrometer and detected with a liquid nitrogen cooled charge-coupled device (CCD) camera. Measurements were carried out in the spectral range from 200 to $1050 \mathrm{~nm}$ (6.2-1.18 eV). To take spectra with high resolution, this spectral range was separated into several parts. Each part of the spectrum was projected onto a CCD matrix containing $1024 \times 256$ pixels.

If the size of the nanoparticles is much smaller than the curvature radius of the tip, and their concentration is large, as in our case, the contrast of the STM image is not clear, i.e. gaps between individual nanoparticles couldn't be fully resolved. Hence, the same Au nanoparticle film was also prepared on a copper transmission electron microscopy mesh with diameter of $3 \mathrm{~mm}$ with a carbon film with thickness of about $10 \mathrm{~nm}$ that is transparent to an electron beam with energy of $200 \mathrm{keV}$ for further studies in a transmission electron microscope "FEI Tecnai 20 F". 


\section{RESULTS AND DISCUSSION}

Fig. 1 shows the photon emission spectrum in the STM experiment at the applied to the tip voltage of $V_{t}=3 \mathrm{~V}$ and corresponding tunnel current $I=1 \mathrm{nA}$. The light emission occurs due to inelastic tunneling of electrons from the $\mathrm{W}$ tip to the $\mathrm{Au}$ nanoparticle. It is accompanied with excitation of a tip-induced plasmon $[19,20]$. This is a result of the strong electromagnetic interaction between the tip and the nanoparticle causing collective electron oscillations of their coupled electron gases. The decay of the tip-induced plasmon gives a peak at $1.62 \mathrm{eV}$ in Fig. 1, whose energy position is in a good agreement with the literature [7, 8, 14]. This peak remains when the voltage applied to the tip switches from negative to positive.

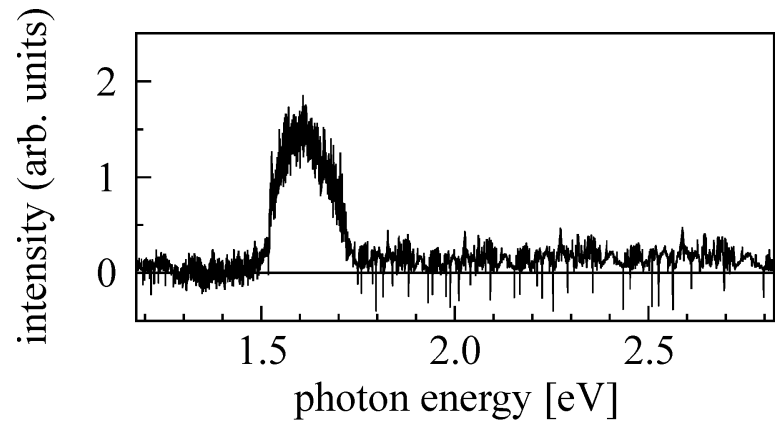

Fig. 1 - Photon emission spectrum of $\mathrm{Au}$ particle (with size about $5 \mathrm{~nm}$ and spherical shape) taken at $V_{t}=3 \mathrm{~V}, I=\mathrm{nA}$

The field emission mode of the STM sets in at increasing of the negative voltage on the tip. The photon emission spectrum in this case is different (Fig. 2, upper curve). In this measurement $V_{t}=10 \mathrm{~V}$ and $I=10 \mathrm{nA}$. This spectrum disappears at a positive polarity current on the tip in contrast to the spectrum in Fig. 1. The tip position was the same for measurements shown in Figs. 1 and 2 (upper curve). The lower curve in Fig. 2 shows the photon emission spectrum exclusively from the pure substrate. This measurement was performed in the STM in the field emission mode at moving the tip to a substrate area that was not covered by nanoparticles. The spectra were decomposed (deconvoluted) as shown in Fig. 2. The result is good enough when Gaussian curves are used for the decomposition. As one can see there are four peaks at energies 1.45, $1.69,1.98$ and $2.22 \mathrm{eV}$ on the upper curve, while the lower one consists only from two peaks at 1.69 and $1.98 \mathrm{eV}$. The energy position of the 1.69 and $1.98 \mathrm{eV}$ peaks are identical for the both curves. We assume that in the field emission mode, the electrons from the tip illuminate the investigated particles as well as partially the substrate. In the tunneling mode this cannot happen due to the protruding nanoparticles. It is possible when the curvature radius of the $\mathrm{W}$ tip is much larger than investigated the Au particles $(R>r)$. After the spectral measurements radius of the tip and size of the $\mathrm{Au}$ nanoparticles were measured using scanning electron microscopy and transmission electron microscopy, respectively. It was obtained that the tip radius $R$ was $60-100 \mathrm{~nm}$ in different cross-sections and the average size of the Au particles $2 r$ was $5 \mathrm{~nm}$.

The peak at $2.22 \mathrm{eV}$ corresponds to excitation and decay of the Mie plasmon in a mode polarized perpendicular to the substrate $[13,14]$ (it was improperly assigned to inter-band transition in the early publications $[2,3])$. Its position on the energy scale is determined by the material, size and shape of the nanoparticles, their concentration and the dielectric constant of the environment.

Another low-energy peak at $1.45 \mathrm{eV}$ is clearly observed but has not been discussed during the analysis of photon emission spectra in systems $\operatorname{tip}(\mathrm{W}) /$ nanoparticle( $(\mathrm{Au}) /$ substrate $\left(\mathrm{TiO}_{2}\right.$ or $\left.\gamma-\mathrm{Al}_{2} \mathrm{O}_{3}\right)$, that were measured in the STM in the field emission mode (see Fig. 2 in Ref. [13] and Fig. 5 in Ref. [14]).

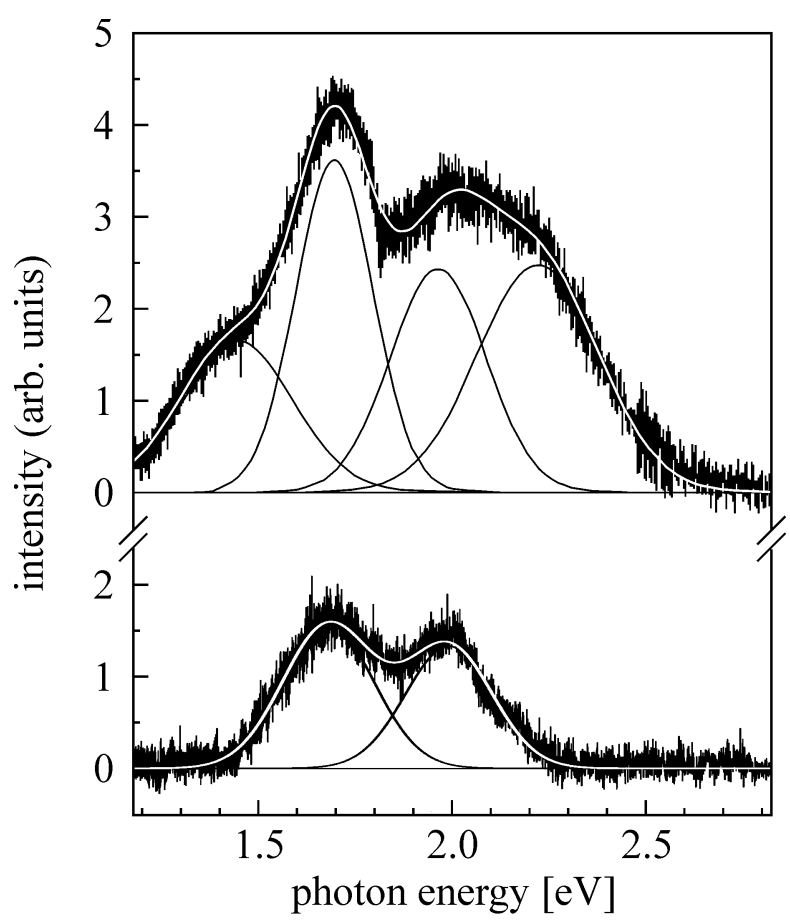

Fig. 2-Photon emission spectra of the same Au particle as in Fig. 1, that is situated on a native oxide covered silicon substrate (upper curve), and of the substrate itself (lower curve) taken at identical experimental conditions $\left(V_{t}=10 \mathrm{~V}\right.$, $I=10 \mathrm{nA})$

Due to the excitation electrons move to the energy levels of the electronic structure of $\mathrm{Au}$ nanoparticles that were unoccupied previously and then make a transition to a state near the Fermi level under the photon emission. This makes the present measurements very informative for the investigation of the electron density of states above the Fermi level.

\section{CONCLUSIONS}

We present experimental evidence of photon emission from Au nanoparticles excited using the STM with $\mathrm{W}$ tip in the tunnel $\left(V_{t}<5 \mathrm{~V}\right)$ and field emission modes $\left(V_{t}>5 \mathrm{~V}\right)$. In the first case the tunnel current has an inelastic component connected with a tip-induced plasmon excitation. The corresponding photon emission is characterized by a maximum at $1.62 \mathrm{eV}$. In the second case the photon emission spectrum is more complicated. The spectrum for an Au nanoparticle obtained after subtraction of photon emission from the substrate (a native oxide covered silicon wafer) is characterized 
by peaks at 2.22 and $1.45 \mathrm{eV}$ connected with the Mie plasmon and the density of unoccupied states above the Fermi level, respectively. The low-energy peak at $1.45 \mathrm{eV}$ has not been discussed in the literature. It was more pronounced than in other publications (see Refs. $[13,14])$ most likely due to the more blunt $\mathrm{W}$ tip in our experiment and consequently the larger applied voltage

\section{REFERENCES}

1. J.K. Gimzewski, B. Reihl, J.H. Coombs, R.R. Schlittler, Zeitschrift für Physik B 72, 497 (1988).

2. R. Berndt, J.K. Gimzewski, P. Johansson, Phys. Rev. Lett. 67, 3796 (1991).

3. R. Berndt, J.K. Gimzewski, Surf. Sci. 269-270, 556 (1992).

4. R. Berndt, J.K. Gimzewski, R.R. Schlittler, Ultramicroscopy 42-44, 355 (1992).

5. N.L. Schneider, R. Berndt, Phys. Rev. B 86, 035445 (2012).

6. G. Hoffmann, J. Kliewer, R. Berndt, Phys. Rev. Lett. 87, 176803 (2001).

7. Z.C. Dong, X.L. Zhang, H.Y. Gao, Y. Luo, C. Zhang, L.G. Chen, R. Zhang, X. Tao, Y. Zhang, J.L. Yang, J.G. Hou, Nat. Photonics 4, 50 (2010).

8. T. Wang, E. Boer-Duchemin, Y. Zhang, G. Comtet, G. Dujardin, Nanotechnology 22, 175201 (2011).

9. A. Olkhovets, S. Evoy, H.G. Craighead, Surf. Sci. 453 , L299 (2000).

10. A. Downes, M.E. Welland, Appl. Phys. Lett. 72, 2671 (1998).

11. N. Nilius, N. Ernst, H.-J. Freund, Phys. Rev. Lett. 84, 3994 (2000). (the Au nanoparticle size was in the range of a few nanometers in all cases).

The use of an STM in the field emission mode with the light signal detection allows to implement lowenergy electron-photon spectroscopy (inverse photoemission spectroscopy).

12. N. Nilius, A. Cörper, G. Bozdech, N. Ernst, H.-J. Freund, Prog. Surf. Sci. 67, 99 (2001).

13. N. Nilius, N. Ernst, H.-J. Freund, Surf. Sci. 478, L327 (2001).

14. N. Nilius, N. Ernst, H.-J. Freund, Phys. Rev. B 65, 115421 (2002).

15. R. Berndt, R. Gaisch, J.K. Gimzewski, B. Reihl, R.R. Schlittler, W.D. Schneider, M. Tschudy, Science 262, 1425 (1993).

16. N.L. Schneider, J.T. Lü, M. Brandbyge, R. Berndt, Phys. Rev. Lett. 109, 186601 (2012)

17. P.G. Borziak, Yu.A. Kulyupin, S.A. Nepijko, V.G. Shamonya, Thin Solid Films 76, 359 (1981).

18. S.A. Nepijko, D. Kutnyakhov, S.I. Protsenko, L.V. Odnodvorets, G. Schönhense, J. Nanoparticle Res. 13, 6263 (2011).

19. P. Johansson, R. Monreal, P. Apell, Phys. Rev. B 42, 9210 (1990).

20. B.N.J. Persson, A. Baratoff, Phys. Rev. Lett. 68, 3224 (1992). 